\title{
Heart Rate Variability Of Moscow Secondary Schoolboys Living In Different Environmental Conditions
}

\author{
Marya Danacheva ${ }^{1}$, Viktor Glebov ${ }^{2,1, *}$, Vladimir Torshin ${ }^{1}$, Vasily Shevtsov ${ }^{1}$, \\ Dilyara Efremova $^{3}$, Elizaveta Anikina ${ }^{1}$, and Md Shamsher Alam ${ }^{4}$ \\ ${ }^{1}$ Peoples Friendship University of Russia (RUDN University), Faculty of Ecology, 6 Miklukho- \\ Maklaya Street, Moscow, 117198, Russian Federation \\ ${ }^{2}$ Russian State Agrarian University - Timiryazev Moscow Agricultural Academy, Moscow, 127550, \\ Russian Federation \\ ${ }^{3}$ Russian State University for the Humanities, Moscow, 125993, Russian Federation \\ ${ }^{4}$ Interactive Research and Development (IRD), Dhaka 1212, Bangladesh
}

\begin{abstract}
The presented empirical study assesses the state of the cardiovascular system of Moscow schoolchildren living in different environmental conditions. 233 practically healthy schoolchildren (109 boys and 124 girls aged 11.4 to 12.6 years) took part in the comprehensive study of HRV and the state of adaptive processes when living in different ecological conditions of the capital megapolis. On the basis of different ecological and social conditions the schoolchildren were divided into 4 compared groups. The comparative analysis of the obtained HRV data of schoolchildren of different groups showed differences in the adaptation processes and in the indices of the wave activity of the sympathetic and parasympathetic departments of the ANS.
\end{abstract}

\section{Introduction}

Recently, an important aspect in the development of the health of the younger generation is associated with environmental degradation, which has a significant impact on the development of the neuropsychiatric sphere of children and adolescents during their education in educational institutions [1,2]. Researchers have noted an increase in psychosomatic deviations in the child population, which in turn affects the difficulties in mastering school material [3].

An important role in the process of individual development and adaptation of the body to different environmental conditions is played by the cardiovascular system (CVS), which is sensitive to all changes in the external environment [4,5]. Based on this, our goal was to study the effects of a complex of environmental factors of a large city on the psych functional systems (nervous and cardiovascular) and the adaptation processes of the school population in the context of educational activities.

\footnotetext{
*Corresponding author: vg44@mail.ru
} 


\section{Materials and methods}

Studies of psych functional systems and the state of adaptation of the school population were carried out on schoolboys of secondary schools in Moscow, who lived in different social and environmental conditions of the metropolitan metropolis (Fig. 1.)

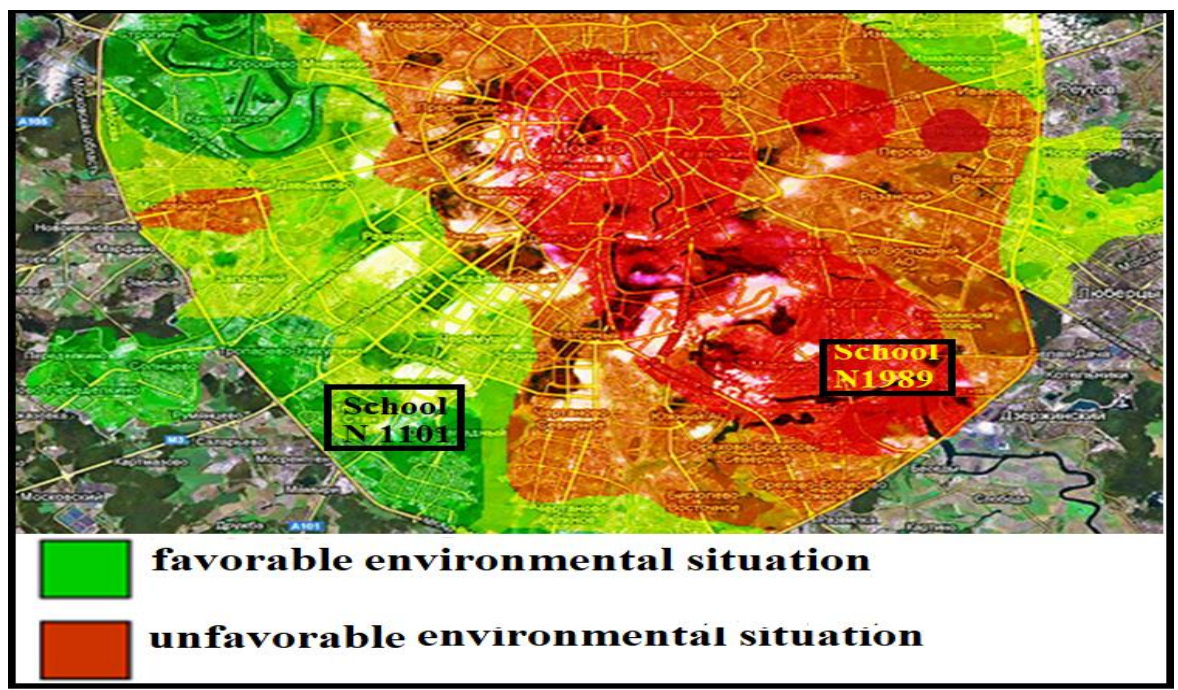

Fig. 1. Location of the studied schools on the territory of Moscow

The total sample of the study was 233 practically healthy schoolboys (109 boys and 124 girls aged 11.4 to 12.6 years).

According to official sources (State reports on environmental monitoring and the sanitary and epidemiological state of the territory of Moscow), it can be noted that school No. 1101 was located on the territory where the environmental situation was considered favorable (a large array of forest zones, urban parks, the absence of industrial production) [6]. At the same time, some families in this sample had a low socio-economic status (low family income, conflict in family relations, poor housing conditions, etc.).

According to official data, the territory where school No. 1989 was located was not in a favorable environmental situation (high atmospheric and soil pollution, the presence of a large number of various industrial enterprises) [6]. Considering the socio-economic status of this sample, it should be noted that it was at a satisfactory level (sufficient family income, harmony in family relations, satisfactory housing conditions, etc.).

Based on the above-listed environmental living conditions of the studied sample of schoolchildren, we divided the schoolboys into four compared groups. The first group was defined as an "unfavorable group" (UG) with unfavorable environmental and social living conditions. The second group was identified as a "transit group 1" (TG1) with unfavorable environmental and favorable social conditions. The third group is the "transit group 2" (TG2), which has favorable environmental and unfavorable social conditions and the fourth group is the "favorable group" (FG), which has favorable environmental and social conditions.

To assess the work of the heart rate variability of the studied schoolchildren, a certified hardware and software complex "Psychophysiologist" (LLC "Medikom", Taganrog) was used.

According to the generally accepted standards of the cardio logical scientific society, the following heart rate indicators were analyzed [7]:

- Heart rate (beats. min) - heart rate-statistical characteristic of the dynamic series of 
cardio intervals;

- HF-the power of high-frequency oscillations in the range of $0.4-0.15 \mathrm{~Hz}$, reflects the level of activity of the parasympathetic link of regulation;

- LF-the power of low-frequency oscillations of the duration of RR intervals in the range of $0.15-0.04 \mathrm{~Hz}$, reflects the level of activity of the vasomotor center.

VLF - very low frequency, slow waves. Their oscillation frequency is less than $0.04 \mathrm{~Hz}$.

The test of the studied sample by the Kolmogorov-Smirnov criterion showed the normality of the sample distribution (large sample size $\geq 50$ ).

All the data obtained were processed statistically using the Statistica 6.0 package (StatSoft. Inc., USA). The level of differences was considered statistically significant at $\mathrm{p}<$ 0.05 .

The research followed all the ethical principles of conducting research involving people as subjects in accordance with the Helsinki Declaration. Participation as study subjects was voluntary and anonymous. Informed consent was obtained from all participants [7].

\section{Results}

The analysis of the scientific literature shows that currently the most frequently used method in the quantitative assessment of the human cardiovascular system is the method of mathematical analysis of heart rate variability (HRV), which is based on the coordinated work of the sympathetic and parasympathetic divisions of the autonomic nervous system (ANS), the work of which is presented in Figure 2.

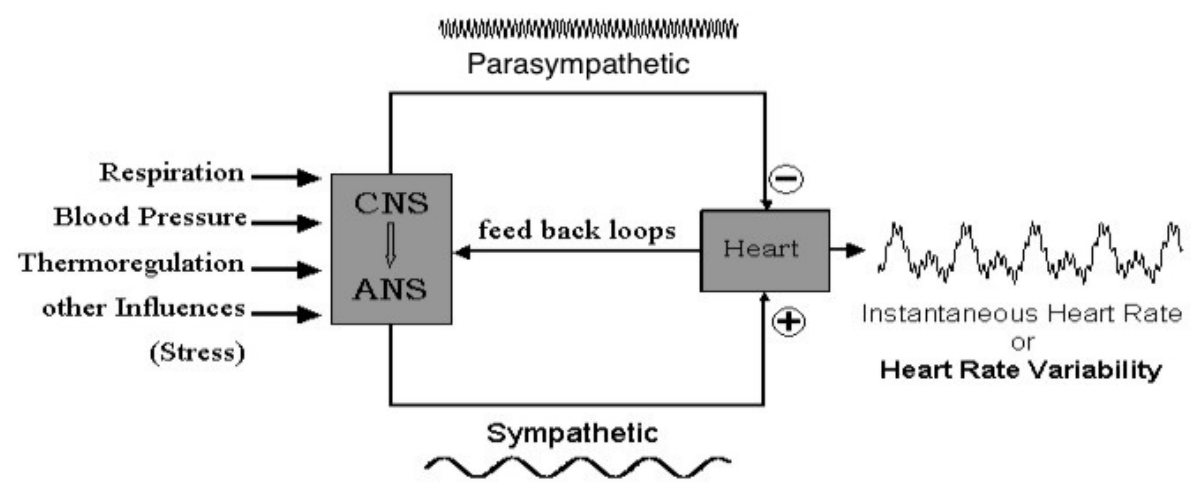

Fig. 2. The mechanism of the autonomic nervous system

The idea of the possibility of using mathematical methods for analyzing the heart rhythm was successfully tested by R. M. Baevsky and O. G. Gazenko [8]. Thus, on the basis of the proposed and widely recognized method (HRV), the spectrum of the heart rate wave activity was studied: high-frequency (HF), low-frequency (LF), and ultra-lowfrequency (VLF) [9].

The analysis of the obtained quantitative data on the HRV of schoolchildren of different UG (E-;S-) and FG (E+;S+) groups revealed the following features: a combination of environmental factors (unfavorable environmental and favorable social environment) led to a sharp activation of the sympathetic division (LF-43\%) and a decrease in the parasympathetic division (HF-28.9\%) in the UG group (43\%). In the other FG group, where a favorable social environment was observed, activity of the parasympathetic HF division was noted: (43\%) and a decrease in the sympathetic division (LF -26\%). The results of the comparative analysis are shown in Figure 3. 

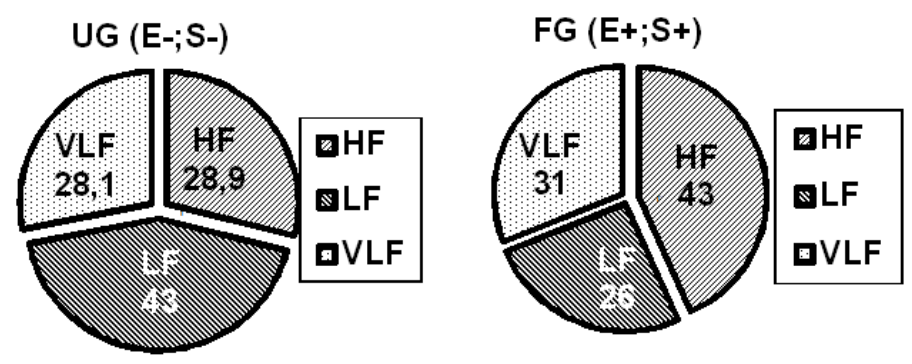

Fig. 3. Distribution of the HRV spectrum of the studied sample of Moscow secondary school schoolboys $(\mathrm{n}=176)$

The distribution of the HRV spectrum in the study sample of Moscow secondary school schoolboys is shown in Figure 4.
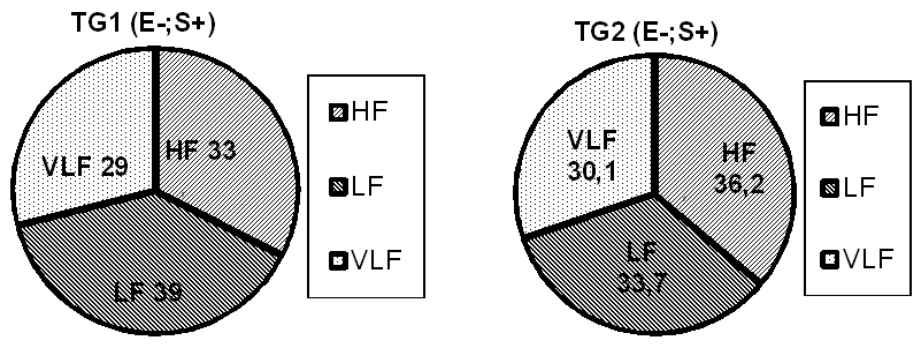

Fig. 4. Distribution of the spectrum of heart rate variability in the study sample of Moscow secondary school schoolboys $(\mathrm{n}=57)$

Comparing the analysis of the TG1 and TG2 groups of the studied sample of secondary school schoolboys with different environmental conditions in terms of HRV revealed the following features: the multidirectional actions of environmental factors in the TG1 group (unfavorable environmental and social environment and vice versa), the wave activity indicators for the sympathetic and parasympathetic parts of the ANS were almost equal. There was no significant excess of the sympathetic division - LF (39\%) and parasympathetic division - HF (33\%) in the TG1 group; In the group of TG2 schoolboys, the same trend is observed: there is no significant excess of the indicators of the sympathetic division - LF (33.7\%) and the parasympathetic division - HF (36.2\%).

An interesting phenomenon in the study of the human cardiovascular system is the data on VLF indicators $[9,10]$. This indicator is associated with the brain activity of an individual, reflecting the adaptive mechanisms of the body of the studied sample of high school schoolboys to the change in the external environment of the metropolitan metropolis. Thus, the VLF values of the $\mathrm{UG}(\mathrm{E}-; \mathrm{S}-)$ and $\mathrm{FG}(\mathrm{E}+; \mathrm{S}+)$ groups were 28.1 and 31.0 , respectively, and the TG1 and TG2 groups were 29 and 31, respectively. The data obtained in the course of the study show complex problems of the adaptation processes of the UG group and partially TG1 and TG2 of the studied schoolchildren living in different environmental conditions, associated with the significant impact of complex factors that have a significant impact on the functional state of the HRV of the studied sample.

\section{Conclusions}

Thus, the study of the complex influence of environmental factors (unfavorable 
environmental and social environment) on the children's body of the UG group showed a significant decrease in the adaptation of secondary school schoolboys, which was expressed in the activation of the sympathetic department (LF-43\%) and a decrease in the parasympathetic department (HF-28.9\%). Under the combined influence of a favorable environmental and social environment on the children's body of the FG group, there was an activation of the parasympathetic division, the activity of the parasympathetic division HF: $(43 \%)$ and a decrease in the sympathetic division (LF -26\%).

With different combinations of unfavorable and favorable effects of the environmental and social environment in the TG1 group, the wave activity indicators for the sympathetic and parasympathetic parts of the ANS showed some differentiated difference: LF (39\%) and HF (33\%); in the TG2 group, the same trend: not a significant excess of the sympathetic part - LF (33.7\%) and parasympathetic part - HF (36.2\%).

An interesting indicator in the assessment of the human cardio system is the VLF indicator. This indicator is associated with the brain activity of an individual, reflecting the adaptive mechanisms of the body of the studied sample of high school schoolboys to the change in the external environment of the metropolitan metropolis. Thus, the VLF values of the UG (E-;S-) and FG (E+;S+) groups were 28.1 and 31.0, respectively, and the TG1 and TG2 groups were 29 and 31, respectively. The data obtained in the course of the study show that the complex problems of the adaptation processes of the UG group, partially TG1 and TG2 of the studied schoolchildren living in different environmental conditions, associated with the significant impact of complex environmental factors, had a significant impact on the functional state of the HRV of the studied sample.

\section{References}

1. B. I., Laver, V. V. Glebov, RUDN Journal of Ecology and Life Safety, 5, 68-73 (2013)

2. V.V. Glebov, G.G. Arakelov. Psychology in Russia: State of the Art. 3, 565-578 (2010).

3. M. N. Danacheva, V. A. Nazarov, V. V. Glebov, The world of science, culture, and education, 6(1), 90-92. (2010)

4. J.T. Bigger et al., J. Am. Coll. Cardiol. 14, 1511-1518 (1989)

5. State reports "On the state and environmental protection of the Russian Federation in 2010-2016»

6. M. Malik, Eur. Heart J., 17, 354 (1996)

7. R. M. Baevsky, O. I. Kirillov, S. Z. Kletskin, Mathematical analysis of changes in the heart rate under stress (Nauka, Moscow, (1984)

8. M. Pagani et al., Circulation, 83 (2), 1143- 1151 (1991)

9. J. Sztajzel, Swiss med wkly, 134, 514-522 (2004)

10. V. I. Torshin, V. R. Gorst, I. N. Polunin, Aghajanyan's readings. Materials of the III All-Russian Scientific and Practical Conference with international participation. Peoples ' Friendship University of Russia, 218-219 (2020) 